\title{
Fixed-Duration Versus Until Disease Progression: How Long Should Initial Treatment of Multiple Myeloma Last?
}

Presented by Yvonne A. Efebera, MD, MPH, and Nina Shah, MD

\begin{abstract}
The number of approved regimens for multiple myeloma has increased dramatically in recent years, improving progression-free and overall survival while also increasing the complexity of treatment decisions. Despite the plethora of options available, one fundamental treatment question remains: How long should initial therapy last? At the NCCN 2019 Annual Congress: Hematologic Malignancies, Drs. Yvonne A. Efebera and Nina Shah debated whether myeloma therapy should be time-limited or continue until disease progression.

J Natl Compr Canc Netw 2019;17(11.5):1430-1432
\end{abstract} doi: 10.6004/jnccn.2019.5033

\section{Continuous Therapy Offers Survival Advantage, But Questions Remain}

In the past 5 years alone, 6 drugs have been FDAapproved for the treatment of multiple myeloma (MM). Despite significant improvements in progression-free survival (PFS) and overall survival (OS), however, questions remain about the appropriate length of initial therapy with these newly approved agents. At the NCCN 2019 Annual Congress: Hematologic Malignancies, Yvonne A. Efebera, MD, MPH, and Nina Shah, MD, presented the merits of fixed-duration versus continuous maintenance therapy for MM. The debate was moderated by Andrew D. Zelenetz, MD, PhD, Memorial Sloan Kettering Cancer Center, and panelists also included Muhamed Baljevic, MD, University of Nebraska Medical Center; Jorge J. Castillo, MD, Dana-Farber Cancer Institute; and Michaela Liedtke, MD, Stanford Cancer Institute.

\section{Set a Fixed Duration}

According to Dr. Efebera, Associate Professor, The Ohio State University Comprehensive Cancer Center - James Cancer Hospital and Solove Research Institute, maintenance therapy improves PFS and OS in patients after autologous stem cell transplantation (ASCT) and in those ineligible for transplantation, but the appropriate duration of treatment is still unknown. In a meta-analysis by Palumbo et $\mathrm{al}^{1}$ comparing continuous versus fixedduration therapy in 3 randomized trials, including a study examining ASCT, patients who received continuous maintenance therapy had a median PFS of 32 versus 16 months for those who received a fixed-duration initial treatment (9 cycles of induction) and no maintenance therapy at all (not even 1 year). "Obviously, there is a benefit for maintenance therapy, but we have yet to determine when enough is enough," said Dr. Efebera. "We just keep going and going until disease progression."

Although no randomized clinical trial has compared maintenance duration (ie, postinduction and postconsolidation), she said, the closest study is a joint effort between the Intergroupe Francophone du Myelome (IFM) trialists group and Dana-Farber Cancer Institute. ${ }^{2}$ In this study, investigators randomly assigned patients with MM to receive either (1) induction therapy with 3 cycles of lenalidomide/bortezomib/dexamethasone (RVD) and then consolidation therapy with either 5 additional cycles of RVD, or (2) high-dose melphalan plus stem cell transplantation followed by 2 additional cycles of RVD. All IFM patients received maintenance therapy with lenalidomide for 1 year, whereas patients enrolled at Dana-Farber received continuous maintenance therapy.

As Dr. Efebera reported, IFM results showed a median PFS of 50 months in the transplant arm, with a 4 -year OS rate of $81 \%$. This is very close to the findings of the meta-analysis of randomized studies of maintenance until disease progression versus placebo, showing a median PFS of 52 months. Results from the United States-based portion of the study that randomized patients to continuous maintenance have yet to be reported.

Toxicity Associated with Maintenance

Although oncologists await data comparing longerduration maintenance versus continuous maintenance 
therapy, providers should consider toxicities associated with maintenance therapy. Data from Attal et $\mathrm{al}^{3}$ showed that more than one-third of patients on lenalidomide maintenance experienced grade $\geq 3$ neutropenia and $58 \%$ had grade $\geq 3$ hematologic toxicity compared with $22 \%$ randomized to placebo. A meta-analysis from McCarthy et $\mathrm{al}^{4}$ demonstrated that nearly $30 \%$ of patients had treatment-emergent adverse events, resulting in them being taken off study. In addition, incidence of a second primary malignancy was $8 \%$ in the maintenance arm versus $3 \%$ in the placebo arm.

Dr. Efebera pointed out that the current monthly cost for lenalidomide exceeds $\$ 8,000$ USD, and because some patients will continue maintenance therapy for up to 7 years, financial toxicity must therefore be considered. A study of quality-adjusted life years (QALYs) that examined the costs associated with maintenance therapy found 5.72 QALYs for the lenalidomide group versus 4.61 QALYs for the no maintenance group. ${ }^{5}$ However, the incremental cost utility ratio exceeded $\$ 300,000$. "This is a high incremental cost-utility ratio impact, which adds uncertainty about maximum duration of treatment," said Dr. Efebera, who emphasized that patients' quality of life is clearly affected while they are on maintenance therapy, with $54 \%$ experiencing cognitive decline. ${ }^{6}$ "Chemo brain is no joke," she emphasized.

\section{The Perfect Study}

In order to provide a definitive answer to the question of therapy duration, Dr. Efebera proposed randomizing patients to 3,5 , or 7 to 10 years of lenalidomide maintenance after 4 cycles of induction and ASCT in transplant-eligible patients or 8 cycles of induction in those who are transplant-ineligible. In the meantime, therapy guided by minimal residual disease (MRD) in a recently initiated SWOG trial could provide some answers (ClinicalTrials.gov identifier: NCT04071457). In this study, patients will receive induction therapy followed by ASCT and will then be randomized to lenalidomide versus lenalidomide/daratumumab. After 2 years of maintenance therapy, patients who are MRD-negative will be randomized to observation versus continuation of treatment in both arms.

"MRD-guided maintenance will hopefully lead us to the path of discovery regarding duration of maintenance for patients with MRD-negative status," Dr. Efebera concluded. "Unfortunately, MRD-positive patients will not be randomized."

\section{Continue Treatment Until Progression}

According to Dr. Shah, Associate Professor, University of California San Francisco, Helen Diller Family Comprehensive Cancer Center, continuous treatment until progression or tolerance is the optimal strategy for patients with MM based on best available evidence. "We know that depth of response is important in MM, and the more you treat, the deeper the response," she said. "This is one of the ideas behind not only transplant consolidation but also maintenance therapy-to potentially increase the proportion of patients who have MRD-negativity, which will then theoretically translate to a greater PFS."

Although the IFM study was not powered to show improvement in OS, CALGB (Alliance) 100104, which assessed lenalidomide versus placebo maintenance after single ASCT for MM, showed improvement in both PFS and OS for patients randomized to the lenalidomide maintenance arm. ${ }^{7}$ According to Dr. Shah, differences in outcomes between the studies may be explained by the "dealer's choice" design of the Alliance trial, which favored increased use of immunomodulatory drugs. Duration of maintenance therapy in the Alliance trial also approached 3 years versus closer to 2 years in the IFM study.

Nevertheless, said Dr. Shah, there are still some unanswered questions with respect to whether maintenance applies to transplant-ineligible versus transplanteligible patients. A meta-analysis of 11 trials of patients with newly diagnosed in the United Kingdom, however, showed improved PFS in the overall population for those receiving lenalidomide maintenance. ${ }^{8}$ Furthermore, a subanalysis of transplant-ineligible patients also showed improved PFS with lenalidomide maintenance versus observation.

According to Dr. Shah, concerns about the harm caused by prolonged lenalidomide maintenance may be unfounded, as well. A whole exosome study of 70 paired presentation relapse samples found no difference in mutational load between patients randomized to lenalidomide maintenance versus observation, she reported. ${ }^{9}$

\section{Duration of Therapy: Longer Still Better}

Because no study has examined the appropriate length of maintenance therapy in a prospective manner, this must be discerned from retrospective data. One analysis compared patients on lenalidomide maintenance for $<12$ months versus 12 to 24 months versus $>24$ months and found that an improvement in PFS was associated with longer duration of therapy. ${ }^{10}$ "These answers are hard to tease out from the data, which of course is always biased, but it looks like receiving longer maintenance therapy is better," Dr. Shah observed.

Despite the PFS and OS advantage, however, there are drawbacks to consider, such as second primary malignancies. "Second primary malignancies are, of course, more prevalent in patients who receive lenalidomide maintenance compared with those who do not," she explained. "This is a really important discussion that we have with all of our patients when they initiate 
maintenance therapy: all good things come with some downsides."

Nevertheless, said Dr. Shah, prolonged lenalidomide maintenance therapy is still the best bet. "When we look across studies, whether thalidomide, lenalidomide, or bortezomib, the favorable aspect is on the side of continuous duration maintenance therapy," she said. "We don't know what continuous means for each individual patient, but continuous seems to be better as far as the OS benefit." Although it is difficult to show improvements in OS because MM is a "marathon disease," she added, providers must do everything they can to make this marathon last as long as possible.

"Continuous therapy has a survival advantage, but ultimately, we have to reconcile this with quality of life," she concluded. "I think the burden is on us as oncologists to design interesting and innovative ways of approaching this problem so that we can optimize therapy for patients and optimize their experiences."

Disclosures: Dr. Efebera has disclosed that she receives honoraria from Janssen Pharmaceutica Products, LP, and Takeda Pharmaceuticals North America, Inc.; and that she is a scientific advisor for Akcea Therapeutics.
Dr. Shah has disclosed that she receives grant/research support from Celgene Corporation, Janssen Pharmaceutica Products, LP, bluebird bio, and Sutro Biopharma, Inc.; owns stock in Indapta Therapeutics; and is a scientific advisor for Genentech, Inc., Seattle Genetics, Inc., Oncopeptides, Karyopharm Therapeutics Inc., Surface Oncology, Precision BioSciences, Inc. GlaxoSmithKline, Nektar Therapeutics, Indapta Therapeutics, and sanofiaventis U.S. LLC. Dr. Zelenetz has disclosed that he received consulting fees from AbbVie, Inc., Amgen Inc., AstraZeneca Pharmaceuticals LP, Celgene Corporation, Gilead Sciences, Inc., Janssen Pharmaceutica Products, LP, Novartis Pharmaceuticals Corporation, Adaptive Biotechnologies

Corporation, Genentech, Inc./Roche Laboratories, Inc., and Pharmacyclics; is a scientific advisor for AbbVie, Inc., AstraZeneca Pharmaceuticals LP, and MorphoSys AG; and receives grant/research support from BeiGene, Gilead Sciences, Inc., MEl Pharma Inc., and Roche Laboratories, Inc. Dr. Baljevic has disclosed that he is a scientific advisor for Karyopharm Therapeutics and Takeda Pharmaceuticals North America, Inc., and receives consulting fees from Cardinal Health and Takeda Pharmaceuticals North America, Inc. Dr. Castillo has disclosed that he receives grant/research support and consulting fees from BeiGene, Janssen Pharmaceutical Products, LP, and Pharmacyclics; and that he receives grant/research support from AbbVie, Inc. and TG Therapeutics, Inc. Dr. Liedtke has disclosed that she receives grant/ research support from Agios, Inc., Amgen Inc., Celator Pharmaceuticals, Genentech, Inc., Gilead Sciences, Inc., Janssen Pharmaceutica Products, LP, bluebird bio, Inc. Prothena, Pfizer Inc., and Takeda Pharmaceuticals North America, Inc; receives consulting fees from Amgen Inc.; and is a scientific advisor for Celgene Corporation, Janssen Pharmaceutica Products, LP, and Jazz Pharmaceuticals Inc

Correspondence: Yvonne A. Efebera, MD, MPH, The Ohio State University Comprehensive Cancer Center - James Cancer Hospital and Solove Research Institute, 320 West 10th Street, A357, Columbus, OH 43210 Email: yvonne.efebera@osumc.edu; and

Nina Shah, MD, University of California San Francisco, 400 Parnassus Avenue, 4th Floor, San Francisco, CA 94143. Email: nina.shah@ucsf.edu

\section{References}

1. Palumbo A, Hajek R, Delforge $M$, et al. Continuous lenalidomide treatment for newly diagnosed multiple myeloma. N Engl J Med 2012; 366:1759-1769.

2. Attal M, Lauwers-Cances V, Hulin C, et al. Lenalidomide, bortezomib, and dexamethasone with transplantation for myeloma. N Engl J Med 2017 . 376:1311-1320

3. Attal M, Lauwers-Cances $\mathrm{V}$, Marit $\mathrm{G}$, et al. Lenalidomide maintenance after stem-cell transplantation for multiple myeloma. N Engl J Med 2012;366:1782-1791.

4. McCarthy PL, Holstein SA, Petrucci MT, et al. Lenalidomide maintenance after autologous stem-cell transplantation in newly diagnosed multiple myeloma: a meta-analysis. J Clin Oncol 2017;35:3279-3289.

5. Olry de Labry Lima A, Gimeno-Ballester V, Ríos Tamayo R, et al. Costeffectiveness of lenalidomide maintenance in patients with multiple myeloma who have undergone autologous transplant of hematopoietic progenitor cells [published online May 31, 2019]. Bone Marrow Transplant. doi: 10.1038/s41409-019-0574-5

6. Stewart AK, Trudel S, Bahlis NJ, et al. A randomized phase 3 trial of thalidomide and prednisone as maintenance therapy after ASCT in patients with MM with a quality-of-life assessment: the National Cancer Institute of Canada Clinicals Trials Group Myeloma 10 trial. Blood 2013; 121:1517-1523.

7. Holstein SA, Jung SH, Richardson PG, et al. Updated analysis of CALGB (Alliance) 100104 assessing lenalidomide versus placebo maintenance after single autologous stem-cell transplantation for multiple myeloma: a randomized, double-blind, phase 3 trial. Lancet Haematol 2017;4:e431-442.

8. Jackson $\mathrm{GH}$, Davies FE, Pawlyn $\mathrm{C}$, et al. Lenalidomide maintenance versus observation for patients with newly diagnosed multiple myeloma (Myeloma XI): a multicentre, open-label, randomised, phase 3 trial. Lancet Oncol 2019;20:57-73.

9. Jackson GH, Davies FE, Pawlyn C, et al. Response adapted induction treatment improves outcomes for myeloma patients; results of the phase III Myeloma XI study [abstract]. Blood 2016;128:Abstract 244.

10. Amsler IG, Jeker B, Mansouri Taleghani B, et al. Prolonged survival with increasing duration of lenalidomide maintenance after autologous transplant for multiple myeloma. Leuk Lymphoma 2019;60: 511-514. 